\title{
Short bar repair of pectus excavatum
}

\author{
Hans Kristian Pilegaard ${ }^{1,2}$ \\ ${ }^{1}$ Department of Cardiothoracic and Vascular Surgery, Aarhus University Hospital, Skejby, Aarhus, Denmark; ${ }^{2}$ Department of Clinical Medicine, \\ Aarhus University, Aarhus, Denmark \\ Correspondence to: Hans Kristian Pilegaard. Department of Cardiothoracic and Vascular Surgery, Aarhus University Hospital, Skejby, Palle Juul- \\ Jensens Boulevard 99, DK-8200 Aarhus N, Denmark. Email: pilegaard@dadlnet.dk.
}

Submitted Aug 08, 2016. Accepted for publication Sep 12, 2016.

doi: $10.21037 /$ acs.2016.09.08

View this article at: http://dx.doi.org/10.21037/acs.2016.09.08

\section{Clinical vignette}

A 15 -year-old boy presented with a moderate pectus excavatum (Figure 1). The preoperative $\mathrm{X}$-rays are shown (Figures 2,3). He complained about cosmetics and did not have any physiological limitations. He plays badminton without symptoms and claims that he has the same level of endurance as his peers. Because of clinical suspicions concerning Marfan syndrome, he had an echocardiography, which was normal.

\section{Surgical techniques}

The surgical technique is described in details in the paper: short Nuss bar procedure (1).

\section{Preparation}

An epidural catheter is placed just before surgery for postoperative pain treatment. The operation is done under general anesthesia. The patient is placed to the far right of the operation table with the right arm in front of the head. The field is draped, so there is access in an area from the jugular notch on the sternum to the umbilicus and laterally from side to side.

\section{Exposure}

The $5 \mathrm{~mm}$ scope port is inserted laterally on the right side at the level of the nipples. The deepest point under the pectus is identified and may be marked outside on the skin in the direction of the scope. The points where the bar should penetrate the chest wall are marked, and may be checked by the thoracoscope if they are in the line with the deepest point by pressing on the point on the skin on the right side, while looking through the thoracoscope. A template of an appropriate length is bent with some overcorrection, and a pectus bar with the same length is bent in the same shape. The ends of the bar are marked on the skin.

\section{Operation}

Small skin incisions $1-2 \mathrm{~cm}$ long are made according to the markings on the skin, and the subcutaneous tunnels are prepared. The introducer is inserted through the right incision and passed through the chest wall at the defined point. The tunnel below the sternum is prepared by blunt dissection with the tip of the introducer, with simultaneous visualization through the thoracoscope. When the tip is at the left marking, it is passed through the chest wall again and pushed to the left through the left subcutaneous tunnel.

The introducer is lifted and the pre-correction is done by manually pushing on the anterior chest wall, especially on the curvatures. A suture or a tape is inserted in the eyelet of the introducer and after retraction of the introducer, is used to guide the bar through the chest. The left end of the bar may be guided by the fingertip through the chest wall. The bar is rotated by using one or two flippers-the instrument which is used to turn the bar. If the right end of the bar is too far away from the chest wall, the bar is flipped back and the end is bent some more, and then flipped back.

A stabilizer is placed on the left side and retracted as medially as possible, and a steel wire around the bar is used to maintain the stabilizer in that position. At this side, the end of the bar may be bent some more using the flipper, but 


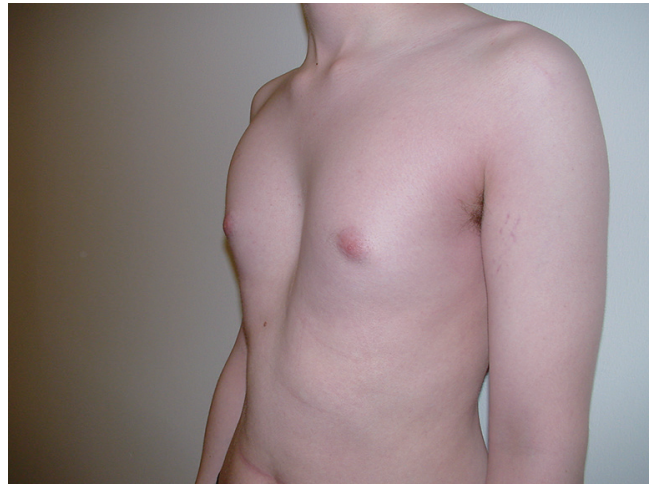

Figure 1 Preoperative picture of the pectus excavatum.

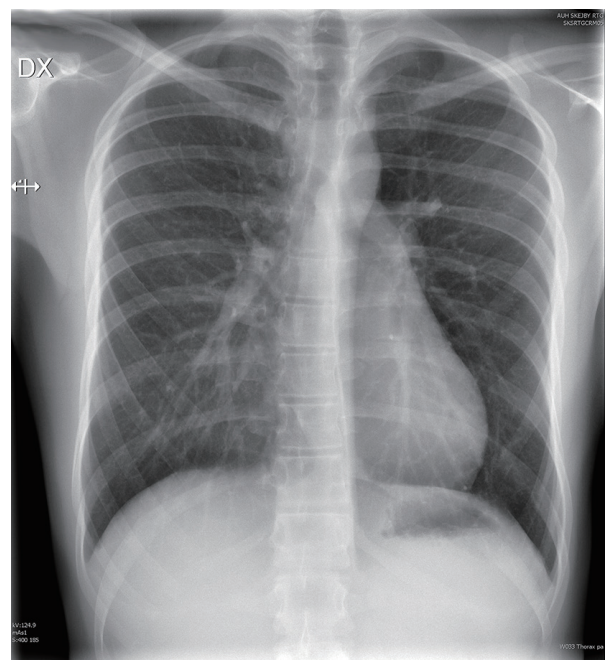

Figure 2 Preoperative $\mathrm{X}$-ray in the A-P position.

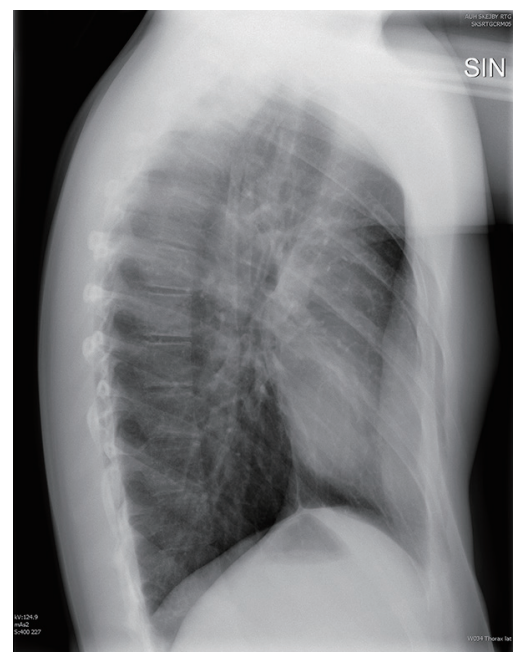

Figure 3 Preoperative X-ray in the lateral position.

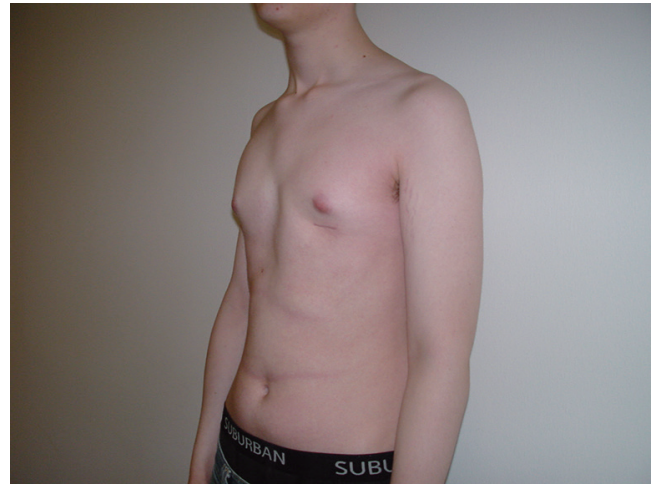

Figure 4 Showing the result after correction of the pectus excavatum.

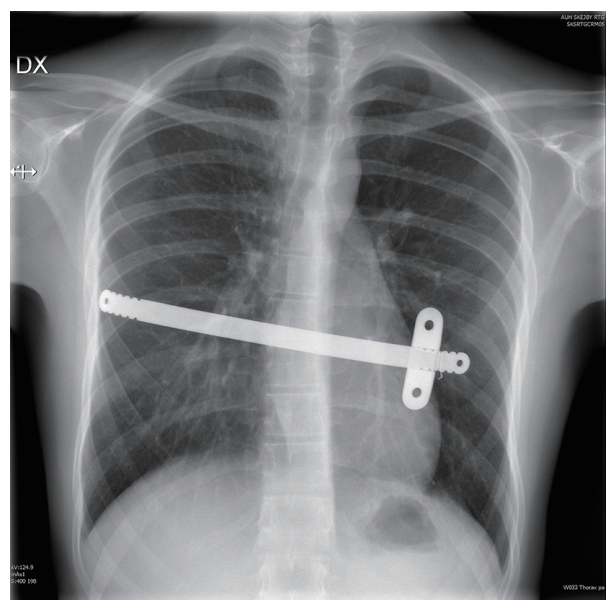

Figure 5 Postoperative $\mathrm{X}$-ray in the A-P position showing the oblique bar.

only after the stabilizer has been placed.

The tunnel under the sternum is checked by the thoracoscope to verify hemostasis and that there are no injuries to the lung parenchyma. A $16 \mathrm{Fr}$. Chest tube is then inserted through the trocar, which is then removed. The anesthesiologist ventilates with some positive-end expiratory pressure (PEEP) to expand the lung, while the skin incisions are closed.

Finally, the ventilation is done by hand with the chest tube connected to an underwater seal to exsufflate the chest cavity. The chest tube is removed and the incision is closed by a steristrip. The surgical steps of the correction of the pectus excavatum are presented in the video (Video 1).

The clinical result 6 weeks postoperatively is shown in Figure 4 and the X-rays in Figures 5,6. 


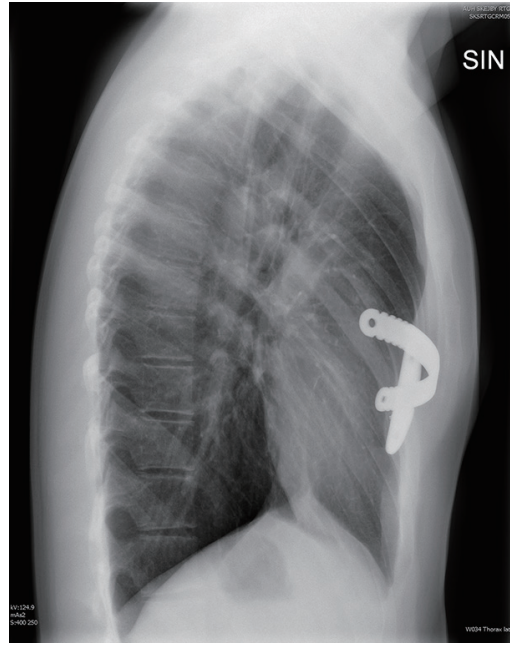

Figure 6 Postoperative X-ray in the lateral position with the bar in situ.

\section{Comments}

Our clinical results have shown that this technique provides a very stable bar with only few cases of bar flipping and dislocation. The median duration of the operation is 36 minutes and less than 1 hour in most patients. The cosmetic results are good with very high rates of patient satisfaction.

\section{Caveats}

If the bar does not pass through the chest wall medial to the highest point, there is an increased risk of stripping of the intercostal muscle. If stripping should happen, the stabilizer should be placed on that side. If you cannot feel the backside of the chest wall, you need to change the introducer to one with a longer tip. The standard introducer that I use is the large size.

If the deepest point of the pectus excavatum lies between two intercostal levels, you will not catch this point with a bar going straight across the chest; instead, the bar may be placed obliquely by using two different levels for entry and exit of the chest wall.

When the pectus is very deep, I prefer to place the first introducer higher than the deepest point. This lifts the deepest point by $3-4 \mathrm{~cm}$, which facilitates the insertion of the next introducer under the deepest point.

If two bars are necessary and should stay close to each other, meaning only one rib apart, they may be inserted through the same skin incisions, but this will not allow you to have two introducers in the patient at the same time. In this situation, you should first place the upper bar and then the lower one. The upper one will in most cases depend too much, so you have to remove it after insertion of the lower one, bend it some more and replace it.

\section{Acknowledgements}

None.

\section{Footnote}

Conflicts of Interest: The author is a Consultant at ZimmerBiomet.

\section{References}

1. Pilegaard HK. Short Nuss bar procedure. Ann Cardiothorac Surg 2016;5:513-8.
Cite this article as: Pilegaard HK. Short bar repair of pectus excavatum. Ann Cardiothorac Surg 2016;5(5):523-525. doi: 10.21037/acs.2016.09.08 